\title{
A Model of Spacetime Dynamics with Embedded Quantum Objects
}

\author{
Hans H. Diel \\ Diel Software Beratung und Entwicklung \\ Seestr.102, 71067 Sindelfingen, Germany \\ diel@netic.de
}

Received 18 August 2017

Accepted 28 September 2017

Published 27 October 2017

\begin{abstract}
General relativity theory (GRT) tells us that (a) space and time should be viewed as an entity (called spacetime), (b) the spacetime of a world that contains gravitational objects should be viewed as curved, and (c) spacetime is a dynamical object with a dynamically changing extent and curvature. Attempts to achieve compatibility of GRT with quantum theory (QT) have typically resulted in proposing elementary units of spacetime as building blocks for the emergence of larger spacetime objects. In the present paper, a model of curved discrete spacetime is presented in which the basic space elements are derived from Causal Dynamical Triangulation. Spacetime can be viewed as the container for physical objects, and in GRT, the energy distribution of the contained physical objects determines the dynamics of spacetime. In the proposed model of curved discrete spacetime, the primary objects contained in spacetime are "quantum objects". Other larger objects are collections of quantum objects. This approach results in an accordance of GRT and quantum (field) theory, while coincidently the areas in which their laws are in force are separated. In the second part of the paper, a rough mapping of quantum field theory to the proposed model of spacetime dynamics is described.
\end{abstract}

Keywords: Causal models; spacetime models; discrete spacetime; relativity theory; quantum field theory.

\section{Introduction}

Discussions on any area of physics are hardly imaginable without having some (at least rudimentary) assumptions about the structure of space and time. With most subjects, the models taught by special relativity theory (SRT) and general relativity theory (GRT) are a sufficient basis for the discussions. With quantum theory (QT), the laws of SRT have been a sufficient base to allow enormous progress in this area of physics. Compatibility of QT with GRT has not yet been achieved, but this circumstance apparently did not retard the progress of QT very much.

This is an Open Access article published by World Scientific Publishing Company. It is distributed under the terms of the Creative Commons Attribution 4.0 (CC-BY) License. Further distribution of this work is permitted, provided the original work is properly cited. 
With the authors work towards a causal model of QT that includes quantum field theory (QFT) (see Refs. 1-3), the adoption of the standard spacetime model of QT also has been a sufficient base for the development of the causal model. However, with recent refinements of the model, questions have arisen that the author could not answer purely from the point of view of standard QT nor from the point of view of existing GRT. In addition, the authors work toward causal models (in general) resulted in a model of space and time in which space and time are, in contrast to the assumption of SRT and GRT, not conceptually integrated into "spacetime". The view of interrelated space and time is then restored as a consequence of causality and the specific causal laws of physics. The questions that occur with the authors proposed causal model of QT/QFT have resulted in the proposal for the spacetime model of the present paper.

The description of the proposed model of spacetime is given in terms of a "causal model". In Sec. 2, a (semi-) formal definition of (the authors understanding of) a causal model is given. A major ingredient of GRT is that spacetime is a dynamical object with dynamically changing extent and curvature. A model of spacetime that does not exhibit this dynamics of spacetime evolution ought to be incomplete. Causal models are the best suited for expressing the complete dynamics in an area of physics.

After Sec. 2, causal models are described in general, in Sec. 3, the basic proposed spacetime model is presented. The processes that compose the dynamics of the spacetime model are described in Secs. 4 and 5. These include the emergence of space, the changes in the existing space caused by a single source and the merging and aggregation of space changes caused by multiple sources. As taught by GRT, the dynamics of spacetime is determined by the energy (including masses) distribution of the physical objects within the space. In the proposed model of curved discrete spacetime, the primary objects contained in spacetime are "quantum objects". Other larger objects are aggregations of quantum objects. This approach achieves a type of integration of QT with GRT. Quantum objects are described in Sec. 6.

In the second part of the paper (Sec. 7), a rough mapping of QFT to the proposed model of spacetime dynamics is described.

\section{Causal Models}

The specification of a causal model of a theory of physics consists of (1) the specification of the system state, (2) the specification of the laws of physics that define the possible state transitions when applied to the system state, and (3) the assumption of a "physics engine."

The physics engine represents the overall causal semantics of causal models. It acts upon the state of the physical system. The physics engine continuously determines new states in uniform time steps. For the formal definition of a causal model of a physical theory, a continuous repeated invocation of the physics engine is assumed to realize the progression of the state of the system. 


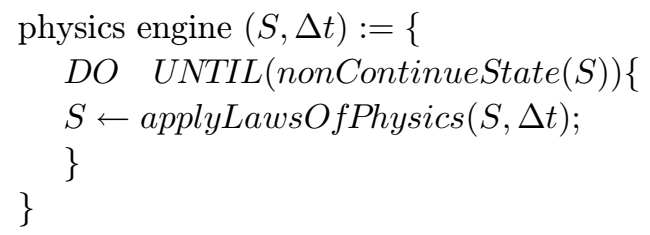

The system state defines the components, objects and parameters of the theory of physics that can be referenced and manipulated by the causal model. In contrast to the physics engine, the structure and content of the system state is specific for the causal model that is being specified. Therefore, the following is only an example of a possible system state specification.

$$
\begin{aligned}
& \text { systemstate }:=\{\text { spacepoint } \ldots\} \ldots \\
& \text { spacepoint }:=\left\{x_{1}, x_{2}, x_{3}, \psi\right\} \\
& \psi:=\left\{\text { stateParameter }_{1}, \ldots, \text { stateParameter }_{n}\right\}
\end{aligned}
$$

The laws of physics: The refinement of the statement $S=$ applyLawsOfPhysics $(S, \Delta t)$; defines how an "in" state $s$ evolves into an "out" state $s$.

$$
\begin{array}{llll}
L_{1}:=I F & c_{1}(s) & T H E N & s \leftarrow f_{1}(s) \\
L_{2}:=I F & c_{2}(s) & T H E N & s \leftarrow f_{2}(s) ; \\
\ldots & & & \\
L_{n}:=I F & c_{n}(s) & T H E N & s \leftarrow f_{n}(s) ;
\end{array}
$$

The "in" conditions $c_{i}(s)$ specify the applicability of the state transition function $f_{i}(s)$ in basic formal (e.g. mathematical) terms or refer to complex conditions that then have to be refined within the formal definition.

The state transition function $f_{i}(s)$ specifies the update of state $s$ in basic formal (e.g., mathematical) terms or refers to complex functions that then have to be refined within the formal definition.

To enable non-deterministic theories ("causal" does not imply deterministic) an elementary function

RANDOM(valuerange, probabilitydistribution) may also be used for the specification of a state transition function.

The set of laws $L_{1}, \ldots, L_{n}$ has to be complete, consistent and reality conformal (see Ref. 4 for more details).

In addition to the above described basic forms of specification of the laws of physics by $L_{n}:=I F c_{n}(s) T H E N s \leftarrow f_{n}(s)$, other forms are also imaginable and sometimes used in this paper. ${ }^{a}$

Note the following comments on the notation used for the specification of causal models. While in mathematics and in programming languages, the "=" sign is used

a This paper does not contain a proper definition of the used causal model specification language. The language used is assumed to be largely self-explanatory. 
for three different purposes, in this paper, three different notations are used for different purposes:

(1) "=" indicates a relation, as in $a=b$, which reads $a$ equals $b$.

(2) ":=" means "is defined as". For example, spacepoint $:=\left\{x_{1}, x_{2}, x_{3}, \psi\right\}$ means spacepoint is defined as ....

(3) " $\longleftarrow$ " indicates a value assignment. $x \leftarrow y$ means that the value of the expression on the right-hand side $(y)$ is assigned to the item on the left-hand side $(x)$.

This notational distinction is used only in causal model specifications. In the traditional mathematical expressions that occur in this paper, the traditional meaning of the "=" sign is used. The distinction of the three types of "equality" has significant implications for the specification of a causal model. This distinction means that typical mathematical equations, such as $T=\frac{1}{2} m \dot{x}^{2}$ (see below), must not be included unchanged in the laws of physics $L_{i}$. The traditional symmetric equal sign that appears in " $T=\frac{1}{2} m \dot{x}^{2}$ " must be replaced by the asymmetric " $\leftarrow$ ". As a consequence, $T \leftarrow \ldots$ and $\dot{x} \leftarrow$ both cannot occur in a causal evolution step (or even within the whole causal model).

Example 1. A causal model: Many areas of physics can be described by starting with a specific Lagrangian. For a description of the causal relationships, i.e., the evolution of the system state, the equation of motion is the major law. The equation of motion can be derived from the Lagrangian by using the Euler-Lagrange equation.

The Lagrangian for classical mechanics is

$$
\begin{aligned}
& \mathrm{L}=\mathrm{V}-\mathrm{T} \text { with } \\
& V=V(x), T=\frac{1}{2} m \dot{x}^{2} .
\end{aligned}
$$

The Euler-Lagrange equation leads to the equation of motion

$$
m \ddot{x}=\frac{\delta V}{\delta x} .
$$

The specification of the laws of classical mechanics can be given by a list $\left(L_{1}, \ldots, L_{n}\right)$ that distinguishes different cases or by a single general law. The single general law is

$$
\begin{aligned}
& L_{1}:=\text { IF }\left(\text { TRUE) THEN FOR }\left(\text { all Particles } P_{i}\right)\{\right. \\
& \left.P_{i} \leftarrow \text { applyEquationOfMotion }\left(P_{i}\right) ;\right\} \\
& \text { Thus, the system state has to contain } \\
& \text { systemstate }:=\{ \\
& \quad \text { space; } \\
& \quad \text { particles }:=P_{1}, \ldots, P_{n} ; \\
& \quad \text { field } V:=V(x) ; \\
& \quad \text { Particle } P:=\{m, x, \ddot{x}, \dot{x}\} \\
& \}
\end{aligned}
$$




\subsection{Requirements for causal models of spacetime}

For causal models of spacetime, obviously, some notion of space and time must be supported. Ideally, the treatment of space and time would be, as much as possible, compatible with SRT and GRT. However, the view of integrated spacetime as assumed with SRT and GRT is already disturbed by the basic causal model described above. In the causal model, space and time are treated differently. Time and the progression of time is an inherent feature of the physics engine. Time is uniformly and simultaneously progressing for the complete system state. Space is the explicit global object that must be included within the system state. Compatibility with SRT and GRT is achieved by the laws that occur in the causal model, which specify the spatial state changes in relation to the (uniform) time changes.

Spatial causal model: A causal model of a theory of physics is called a spatial causal model if (1) the system state contains a component that represents a space, and (2) all other components of the system state can be mapped to the space. There exist many textbooks on physics (mostly in the context of Relativity theory) and mathematics that define the essential features of a "space". For the purpose of the present paper, a more detailed discussion is not required. For the purpose of this paper and the subject locality, it is sufficient to request that the space (assumed with a spatial model) supports the notions of position, coordinates, distance, and neighborhood.

Example 2. A spatial causal model: A possible type of a spatial causal model is the cellular automaton (CA). The classical CA consists of a $k$-dimensional grid of cells. The state of the CA is given by the totality of the states of the individual cells.

$$
\text { state }:=\left\{s_{1}, \ldots, s_{n}\right\}
$$

With traditional standard CAs, the cell states uniformly consist of the same state components

$$
s_{i}:=\left\{s_{i}^{1}, \ldots, s_{i}^{j}\right\}
$$

Typically, the number of state components, $j$, is 1 , and the possible values are restricted to integer numbers. The dynamical evolution of the CA is given by the "update-function", which computes the new state of a cell and of the neighbor cells as a function of the current cell state.

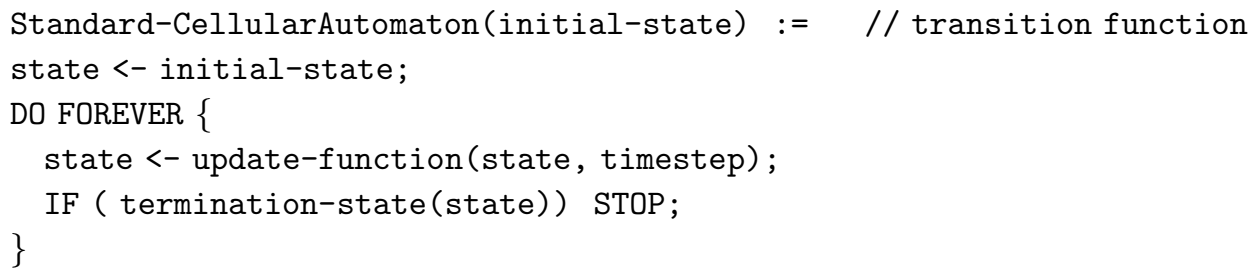

The full functionality and complexity of a particular CA is concentrated in the update-function. As Wolfram (see Ref. 5) and others (see e.g., Ref. 6) have shown, a 
large variety of process types (e.g., stable, chaotic, pseudo-random, and oscillating) can be achieved with relatively simple update-functions. In Ref. 3 a "LagrangianDriven Cellular Automaton Supporting Quantum Field Theory" is described in form of a causal model. In Ref. 7 a CA-based interpretation of quantum mechanics (without reference to causal models) is described.

Local causal model: The definition of a local causal model presupposes a spatially causal model (see above). A (spatially) causal model is understood to be a local model if changes in the state of the system depend on the local state only and affect the local state only. The local state changes can propagate to neighboring locations. The propagation of the state changes to distant locations; however, they must be always accomplished through a series of state changes to neighboring locations. ${ }^{\mathrm{b}}$

Based on a formal model definition of a causal model, a formal definition of locality can be given. We are given a physical theory and a related spatially causal model with position coordinates $x$ and position neighborhood $d x$ (or $x \pm \Delta x$ in case of discrete space-points).

A causal model is called a local causal model if each of the laws $L_{i}$ applies to no more than a single position $x$ and/or to the neighborhood of this position $x \pm d x$.

In the simplest case, this arrangement means that $L_{i}$ has the form

$$
L_{i}: I F c_{i}(s(x)) T H E N s^{\prime}(x)=f_{i}(s(x)) \text {; }
$$

The position reference can be explicit (for example, with the above simple case example) or implicit by reference to a state component that has a well-defined position in space. References to the complete space of a spatially extended object are considered to violate locality.

Multiple asynchronous physics engines to establish units of simultaneity: The requirement of SRT and GRT that differing proper times are possible within a system can be satisfied by the causal model by the assignment of different physics engines with differing proper times to units of simultaneity. In the causal model of QT/QFT described in Refs. 1-3 and in the model of spacetime described in this paper, the units of simultaneity are the "quantum objects". All state changes within the quantum object are considered to occur simultaneously in steps according to the quantum objects proper time. These objects include quantum objects that consist of multiple particles.

Support of indeterminism of QT/QFT: The support of the quantum objects in the causal model of spacetime requires the support of a major feature of QT, namely, indeterminism. As a basis for the support of nondeterministic causal models, the "RANDOM()" function (see above) can be used to specify probability distributions for the outcome of nondeterministic causal developments. For the specification of the laws of QT that concern the development of probability amplitudes, additional support could be useful.

\footnotetext{
b Special relativity requests that the series of state changes does not occur with a speed which is faster than the speed of light. This requirement is not considered within the present paper.
} 
Mapping of derivatives to system state components: In the laws of physics that are part of the causal model, references to variables can occur. The variables that occur can be either (1) components of the system state or (2) variables that are local to the conditions $c_{i}(s)$ or the state transition functions $f_{i}(s)$ of a law $L_{i}$. This arrangement leads to the question of how the derivatives, such as $d \psi / d x, d \psi / d t$ which occur in many standard formulations of laws of physics, should be treated. The straightforward answer is that when the respective derivative can be computed (i.e., derived) within the $c_{i}(s)$ or $f_{i}(s)$, there is no reason for making it part of the system state. The author's experience is that, on the other hand, derivatives with respect to time (for example, momentum and speed) typically must be explicit components of the system state. ${ }^{\mathrm{c}}$

\section{The Causal Model of Curved Discrete Spacetime}

In the causal model of curved discrete spacetime, all dynamics (emerging of space, expansion of space, changing of space curvature) is triggered by the dynamics of the quantum objects embedded in the space. This dynamics includes the aggregation of quantum objects to larger objects (e.g., solid material, planets, and stars) and the related aggregation of the gravitational effects.

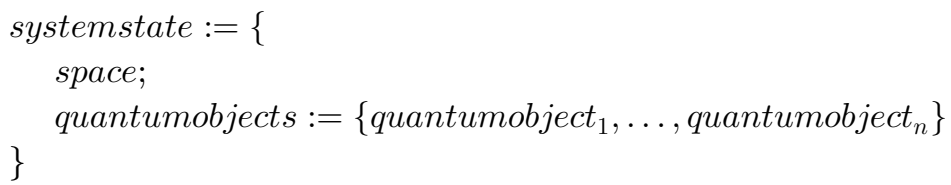

At the highest level, the overall system evolution is specified as follows: systemevolution ()$:=\{$

spaceevolution (space);

FOR (all quantumobjects quantumobject $\left.{ }_{i}\right)\{$

progress (quantumobject $\left.{ }_{i}\right)$;

\}

Because, in general, the quantum objects progress with differing proper time, for the causal model, it is assumed that each quantum object has assigned a separate physics engine (see Fig. 1). A further separate physics engine is assigned to the (global) space. The discrete structure of the spacetime of the model is derived from the causal dynamical triangulation (CDT) (see Refs. 8-10). The total space consists of a collection of space elements.

$$
\text { space }:=\left\{\text { spaceelement }_{1}, \ldots, \text { spaceelement }_{n}\right\}
$$

With CDT, the basic space elements are $n$-dimensional simplexes (e.g., triangles, tetrahedrons) (see Fig. 2). In contrast to CDT, the proposed causal model of curved

\footnotetext{
${ }^{\mathrm{c}}$ Otherwise, the causal model would have to maintain versions of the system state.
} 

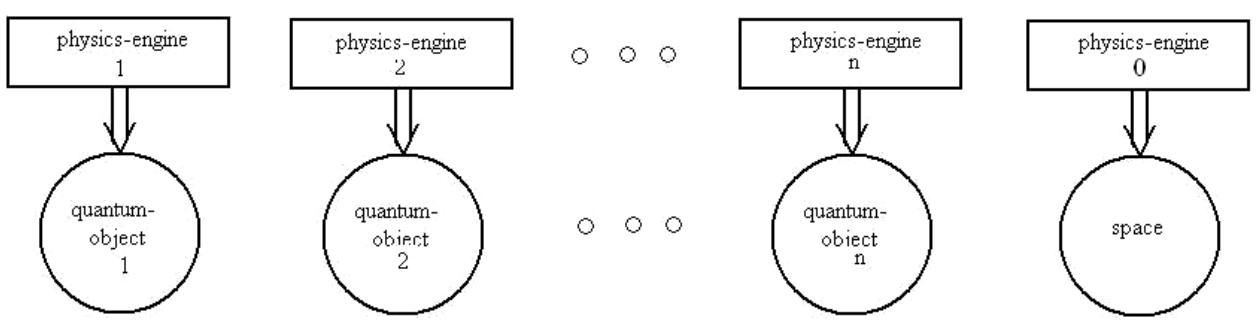

Fig. 1. Physics engines assigned to quantum objects and to space.

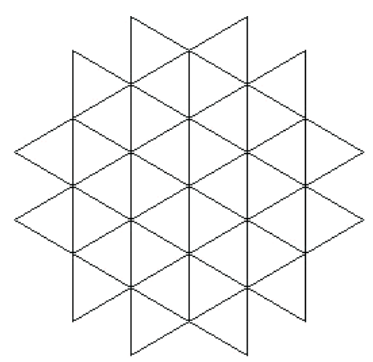

2-dimensional

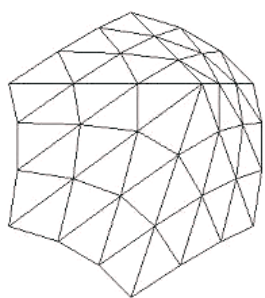

2-dimensional curved

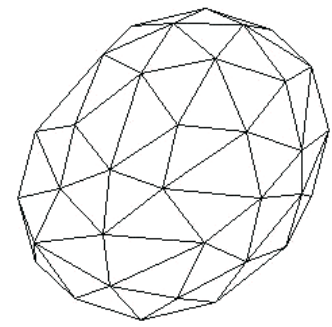

3-dimensional

Fig. 2. Elements of spacetime of causal dynamical triangulation.

discrete spacetime considers only three-dimensional simplexes, i.e., tetrahedrons, because the time dimension is treated separately within the causal model.

\section{Spacetime Emerged from a Single Source}

After Hubble detected that our universe is continuously expanding, physicists concluded that the emergence of spacetime started from some minimal (or zero) spacetime object. The respective event from which the expansion started is called the Big Bang. A model of spacetime dynamics that does not support the possible emergence of spacetime from some minimal spacetime object would therefore be doubtful. The model presented in this paper goes even farther. The model assumes not only that the existence of spacetime originated from a minimal source but also that the subsequent spacetime dynamics originated from events that occurred at minimal elementary objects. In the proposed model, the minimal elementary objects that trigger the spacetime dynamics are the "quantum objects". In Sec. 6 ("Quantum objects as elementary units of causality and locality"), quantum objects are discussed in more detail. 


\subsection{The emergence of space}

The most trivial process for the emergence of space ${ }^{\mathrm{d}}$ might be the following:

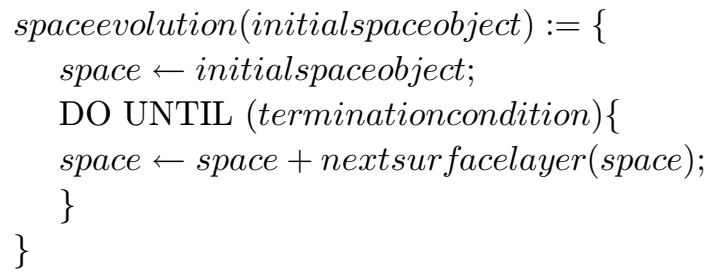

Thereby the function "nextsurfacelayer(space)" however turns out to be nontrivial, if the basic space elements are tetrahedrons and the following conditions/ assumptions are stated:

(1) The space creation starts from some minimal initial collection of space elements (not necessarily from a single element)

(2) Space expansion steps are performed by the physics engine in uniform time steps according to the proper time associated with the space.

(3) The space is always (i.e., after each expansion step) a compact object. There are no holes within this object, and there always exists a surface. The topology of the space surface represents a sphere that consists of a finite number of triangles.

(4) Space expansion occurs in the form of layers. Each expansion step adds another layer of space to the existing space.

(5) The space expansion layers (and thus the total space) consist of a (discrete) number of uniform space elements (e.g., tetrahedrons).

As will be discussed below, the major problem is caused by requirement (5).

For the design of a space expansion algorithm that satisfies the above listed conditions/assumptions, the first question to be answered is what should be the initial-space-object from which the emergence of space starts. The simplest solution would be to start with a single space element (i.e., a single tetrahedron, see Fig. 3 case (a)) and apply the general expansion algorithm at each expansion step. However, the author experienced that starting with an asymmetric object such as the single tetrahedron apparently requires a complicated algorithm to develop toward an (almost) symmetrical spherical space object. The next alternative investigated by the author was the double-tetrahedron, which is at least more symmetrical than the single tetrahedron (see Fig. 3 case (b)). This approach enabled a simpler, yet still nontrivial expansion algorithm. Ultimately, the third attempt, which started with a 12-pack of tetrahedrons (see Fig. 3 case (c)), which is symmetrical in the three perpendicular axes, enabled a somewhat simple expansion algorithm. The conclusion drawn by the

\footnotetext{
d The author uses the formulation "emergence of space" rather than "emergence of spacetime" because the causal model relates the emergence of space to the assumed uniform progression of time.
} 


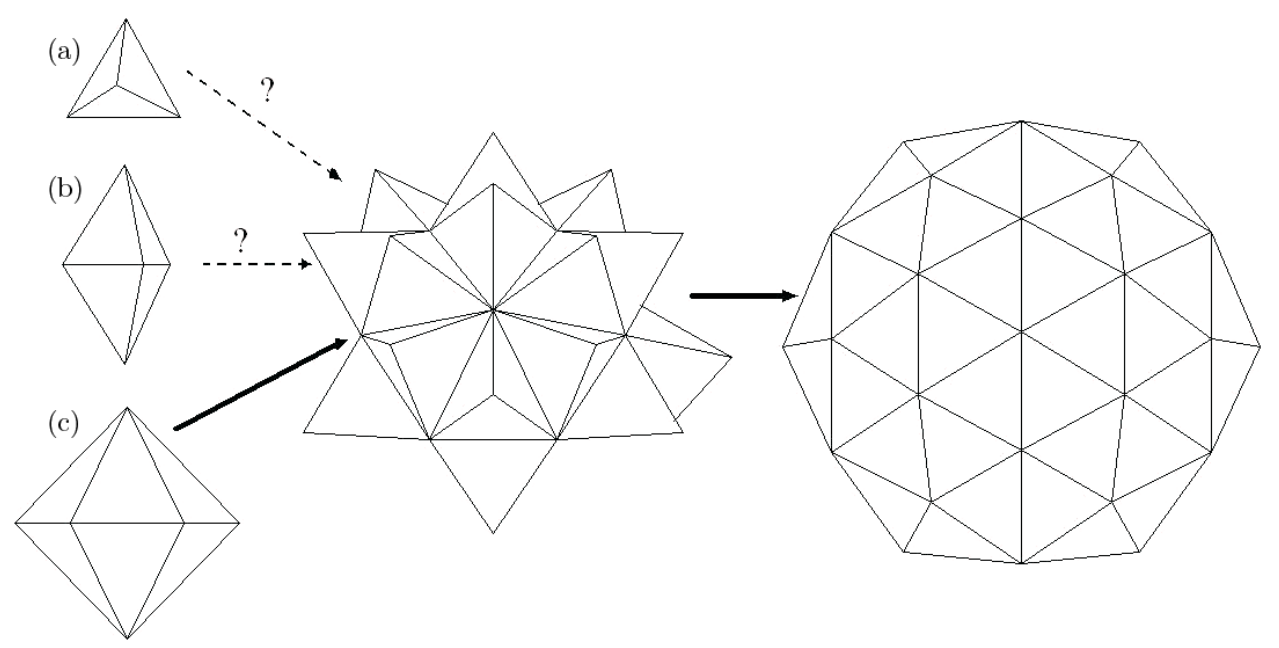

Fig. 3. Emergence of space from triangulated space elements.

author for the determination of the initial space object is that we probably must choose a model in which one of the following is the case:

(1) Only a special highly symmetrical initial-space-object is supported (such as case (c)), or

(2) A variety of initial-space-objects is possible, and a somewhat complicated expansion algorithm is assumed such that the initial-space-object evolves toward a symmetrical (e.g., spherical) space object, or

(3) A variety of initial-space-objects is possible, and the resulting large-scale space object could also be asymmetrical. Asymmetries in the later large-scale space object (i.e., the later universe) could be eliminated by the space dynamics caused by multiple sources (see Sec. 5.2).

The next question to be answered for the design of a space expansion algorithm is to what extent requirement 5 above (the goal to have uniform space elements) can be satisfied. With CDT (see Ref. 11), perfectly identical regular tetrahedrons are assumed. The author has not been successful in designing a space expansion algorithm that uses exactly identical regular tetrahedrons. Assume that in an expansion step the space is uniformly extended by a layer with height $h$. This would mean, that for a space object with (almost) constant shape, the radius of the space object grows linearly from $h$ to $n^{*} h$. The surface of the space object, which consists of an integral number $k$ of uniform triangles must grow as the square of $n$.

$$
n \rightarrow n+1: \text { radius }=h * n \rightarrow h *(n+1) ; k=a_{n} * n^{2} \rightarrow a_{n+1} *(n+1)^{2}
$$

An algorithm that delivers integral numbers $a_{i}$ could not be found. Even when a nonuniform factor $a_{i}$ was accepted, the author could not determine an appropriate 
algorithm for mapping of surface $_{n}$ to surface $_{n+1}$, which preserves the size and shape of the tetrahedrons and triangles.

The author's conclusion has been to give up on having a perfect uniform size and shape of the tetrahedrons and accept variations. Given the surrender of uniform space elements, the question arises whether the model of three-dimensional (3D) space elements that have a certain volume may be obsolete. As an alternative, in the following, it is assumed that the constituents of the space are the space points together with the connections between the space points

$$
\begin{aligned}
& \text { Space }:=\{\text { spacepoint } \ldots\} \\
& \text { spacepoint }:=\{\text { connections, spacecurvature, spacecontent }\}
\end{aligned}
$$

and spacecontent represents the field(s) that fill the space. (QFT assumes that even a vacuum contains fluctuating field(s).) Whether the spacepoints together with the connections form a collection of $n$-dimensional space elements depends also on the details of the causal model of the emergence of space. ${ }^{\mathrm{e}}$

\subsection{The representation of space curvature}

In Sec. 4.1 above, the space component of the system state is specified as consisting of a set of spacepoints and at the next level of detail, a spacepoint is specified as consisting of connections, spacecurvature and spacecontent.

spacepoint $:=\{$ connections, spacecurvature, spacecontent $\}$.

Space curvature is a major ingredient of GRT. Because the causal model of spacetime aims for maximum compatibility with GRT, the space curvature is also a major ingredient of the spacetime model. The following discusses how the space curvature could be expressed in such a way that it is suitable to subsume the role that space curvature takes in GRT. In GRT, specifically in Einsteins equation

$$
G^{\alpha \beta}=8 \pi T^{\alpha \beta}
$$

space curvature is expressed by the curvature tensor $G^{\alpha \beta}$. Thus, the simplest solution would be to say that the space curvature component of the spacepoint provides the same information as the curvature tensor of GRT. However, some adaptations appear reasonable. The major argument that suggests adaptations toward the spacetime model that is based on discrete elementary space elements is that the space that emerges from a source in the way described in Secs. 4.1 and 5.1 which can be viewed as already expressed in some type of curvature. The surfaces of the emerging (3D) space objects shown in Figs. 2 and 3 are spherical, which means that they express curvature. The shown curvature is the result of the manner in which the spacepoints are interconnected, which is the result of the way that the space evolves from the

\footnotetext{
e With the detailed causal model presented in this paper, there are a number of aspects that apparently enable the view of 3D (non-uniform) space elements such as tetrahedrons. However, thus far, no argument has been found by the author to insist on uniform space elements.
} 
source(s). With a closer look, however, it becomes clear that the curvature expressed in the geometry of the spacepoint connections alone is not a sufficient substitute for the curvature tensor $G^{\alpha \beta}$ of GRT. Nevertheless, it appears reasonable to consider the network of interconnected spacepoints as the basis curvature specification and to assign additionally required curvature-related information to the spacepoints and/or to the spacepoint connections.

$$
\begin{aligned}
& \text { spacepoint }:=\{\text { connections, spcurvature, spacecontent }\} \text {; } \\
& \text { connections }:=\left\{\text { connection }_{1}, \ldots, \text { connection }_{n}\right\} \text {; } \\
& \text { connection }:=\{\text { targetspacepoint, concurvature, } \Delta \text {.curvature }\} ;
\end{aligned}
$$

The connection-related curvature "concurvature" specifies the geometrical relationships in 3D space. The spacepoint-related curvature "spcurvature" specifies the (physical) strength of the curvature. With the emergence of space and the propagation of space from a single source, spcurvature is proportional to the sources energy (including mass) and decreases with the distance from the source.

A further parameter " $\Delta$.curvature" is required which specifies the propagation of space curvature changes in space (see Sec. 5). If $\Delta$.curvature $=0$, no propagation of space changes is currently active at the respective spacepoint connection (see Sec. 5 for more details).

Mathematically, the curvature of an $n$-dimensional (differentiable) manifold is often equated with the change of a tangent vector that is transported on a surface from point P1 to point P2 following two different paths on the surface. In Fig. 4, the tangent vector transport is shown (a) for a differentiable sphere and (b) for the surface of the triangulated space objects considered in Secs. 4 and 5. Although the surfaces that develop during the spacetime dynamics (Fig. 4(b)) do not represent differentiable manifolds, an equivalent to the tangent vector parallel transport can nevertheless be defined. Let us assume that a path on the triangulated surface must always follow connections between the spacepoints (i.e., the border of a surface

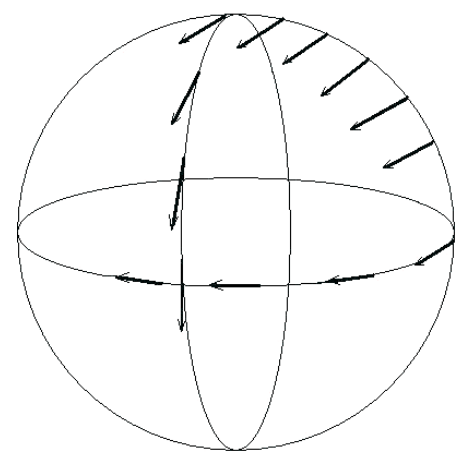

(a)

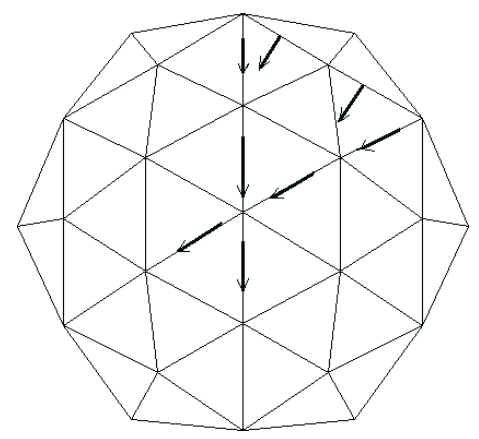

(b)

Fig. 4. Tangent vector parallel transport on a differentiable sphere and on a sphere consisting of triangles. 
triangle) and that the tangent vector is given by its angle relative to the vector that shows the direction of its movement. This arrangement means that when the tangent vector moves from connection- 1 to connection- 2 , it must be rotated according to the angle $\alpha$ between connection- 1 and connection- 2 .

This rule for the tangent vector parallel transport indicates that the relevant parameters that determine the curvature are the angles $\alpha$ between the connections. Therefore, it is reasonable to use the angles $\alpha$ for the specification of the concurvature component. However, note that the angles $\alpha$ are sufficient to specify the curvature of a 2-sphere, but they are not sufficient for the specification of the impact that the gravitational curvature has on the motion of a test particle. For the GRT-compatible causal model of spacetime dynamics additional curvature-related parameters as described above are required.

\section{The Dynamics of Spacetime}

Similar to the emergence of space, the dynamics within existing space is assumed to be triggered by the dynamics of the contained quantum objects. This circumstance means that the space is constantly changing. The major type of change that must be considered (and is considered in the present section) are changes in the space curvature. The most famous example of curvature change is gravitational waves. However, gravitational waves are only a special type of the propagation of curvature changes.

For a discussion of the propagation of space changes, it is useful to treat the space similar to a field, the gravitational field, similar to other fields known in physics. This approach means that the attributes and properties are associated with spacepoints, and the dynamics of the associated attributes/properties must be discussed. In the following, it is assumed that the set of spacepoints does not change, except for a possibly concurrently occurring expansion of the space. The changes affect only the connections between the spacepoints, including the curvature. To support the law of SRT/GRT, which states that actions can propagate within space with the final speed only it is assumed that with an update step of the physics engine of the space, space changes may propagate to neighboring spacepoints only (see local causal models in Sec. 2). In addition, it is assumed that with an update step of the physics engine of the space, the complete space (i.e., all spacepoints that are subject to change) are changed simultaneously.

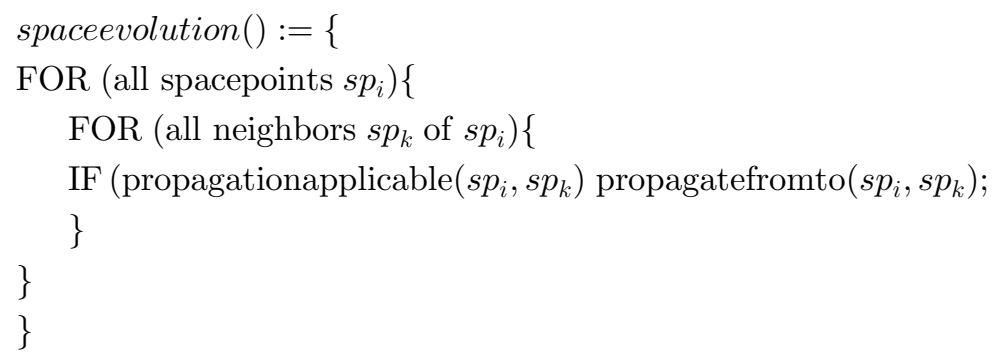


The condition propagationapplicable () and the state transition function propagatefromto() define the details of the spaceevolution(). Both propagationapplicable() and propagatefromto() are asymmetric conditions/functions, i.e.,

$$
\begin{aligned}
& \text { propagationapplicable }\left(s p_{i}, s p_{k}\right) \neq \text { propagationapplicable }\left(s p_{k}, s p_{i}\right) ; \\
& \text { propagatefromto }\left(s p_{i}, s p_{k}\right) \neq \text { propagatefromto }\left(s p_{k}, s p_{i}\right)
\end{aligned}
$$

The asymmetry of propagationapplicable() prevents the occurrence of propagation loops and of mutual causal dependencies. The asymmetry of propagationapplicable() and propagatefromto() implies also that for a given propagation step (of the physics engine of the space) and for a given spacepoint, the connections of the spacepoints can be classified into (1) "in-connections" and (2) "out-connections" (and connections that are neither in-connections nor out-connections). This approach allows a rewrite of specification (4) to

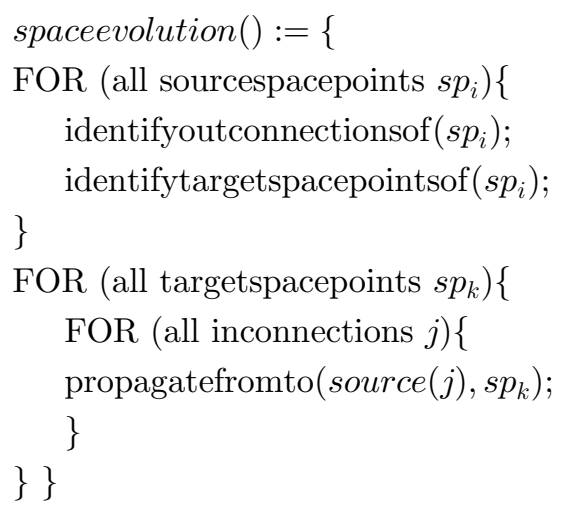

In physics, details of the flow of propagation of changes are typically derived from the equation of motion (which can be derived from the Lagrangian). Typically, the equation of motion contains the second derivatives of the changing property $\psi$. As described in Sec. 2.1, for the specification of a causal model, the derivatives with respect to time (e.g., $d \psi / d t$ ) must usually be mapped to explicit components of the system state. In the causal model of space changes, the derivative of the curvature is explicitly included in the system state in terms of the $\Delta$.curvature component of the connection (see Sec. 4.2).

$$
\text { connection }:=\{\text { targetspacepoint, curvature, } \Delta \text {.curvature }\} \text {; }
$$

$\Delta$.curvature is also the major parameter that determines whether a spacepoint is a source spacepoint or a target spacepoint and whether a connection is an inconnection or an out-connection (relative to a specific spacepoint).

In the literature on GRT (see, for example, [12, Chap. 9]), the propagation of changes in gravitational fields is addressed, however only for special cases, such as weak gravitational fields, distant sources, gravitational waves, among others. The absence of a general treatment causes some reservations against the special cases (e.g., gravitational waves) by some physicists. The author claims that, given the 
extreme dynamics of GRT, a possible general treatment of the propagation of space changes cannot be derived purely from the respective differential equations of GRT together with the local causality condition (i.e., that changes can propagate to neighboring locations only).

As the general schema for the propagation of space changes within the causal model, the following refinement of the function propagatefromto $\left(s p_{i}, s p_{k}\right)$ is specified:

$$
\begin{aligned}
& \text { propagatefromto }\left(s p_{i}, s p_{k}\right):=\{ \\
& s p_{k} . \text { curvature } \leftarrow f 1\left(s p_{i} \cdot \text { curvature }\right) ; \\
& s p_{k} . \Delta . \text { curvature } \leftarrow f 2\left(s p_{i} \cdot \Delta . \text { curvature }\right) ; \\
& s p_{i} . \text { curvature } \leftarrow f 3\left(s p_{i} \cdot \text { curvature }\right) ; \\
& s p_{i} . \Delta . \text { curvature } \leftarrow f 4\left(s p_{i} \cdot \Delta \text {.curvature }\right) ; \\
& \}
\end{aligned}
$$

The detailed functions $f 1(), f 2(), f 3()$ and $f 4()$ remain open. To simplify the description within this paper, the following settings are considered reasonable and are assumed for the remainder of this paper:

$$
\begin{aligned}
& f 1(\text { sp.curvature }):=\text { decreasebyfactor }(\text { sp.curvature, } k) 0<k<1 \\
& f 2(\text { sp. } \Delta . \text { curvature }):=\text { sp. } \Delta . \text { curvature, i.e., unchanged momentum } \\
& f 3(\text { sp.curvature }):=\text { sp.curvature, i.e., unchanged } \\
& f 4(\text { sp. } \Delta . \text { curvature }):=0), \text { i.e., reset }
\end{aligned}
$$

\subsection{The change in the existing space caused by a single source}

In the present section, it is assumed that the source that causes the space changes is a single quantum object. Space changes caused by (large) collections of quantum objects (e.g., bulk matter, planets, and stars) are typically approximated by the assumption of a single source with a center-of-mass. The extent to which such an approximation is reasonable will be discussed in Sec. 5.2.

As part of the evolution of the quantum object, the quantum object periodically initializes a sphere of spacepoints that surround the quantum object with an updated curvature and $\Delta$.curvature. With the next space update step of the physics engine of the space (see spaceevolution() above) the $\Delta$.curvature component of the connection causes the propagation of the curvature change to connected spacepoints. The propagation is continuous with the repetitive invocation of spaceevolution() by the physics engine of the space. The state transition function propagatefromto $\left(s p_{i}, s p_{k}\right)$ performs the propagation of the curvature by continuously weakening the curvature with each propagation step. Here, propagatefromto $\left(s p_{i}, s p_{k}\right)$ also sets the $\Delta$.curva ture component of $s p_{k}$ and reduces the $\Delta$.curvature component of $s p_{i}$ by a factor to be determined.

The change in space caused by a moving source: The changes in space propagate symmetrically from the source in all directions. Because in the causal model of spacetime, the space change requests at the source are emitted periodically with a 

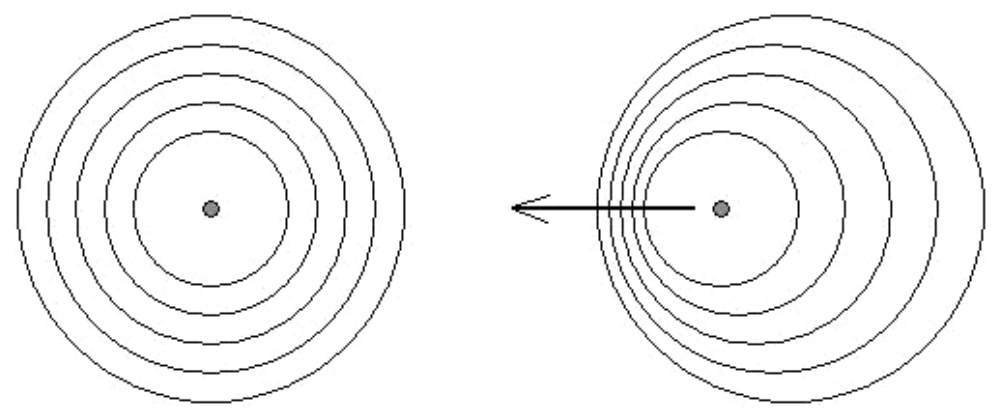

Fig. 5. Propagation of space curvature changes.

frequency determined by the sources proper time and energy (including mass), a continuous series of spherical space curvature changes occurs (see Fig. 5, left-hand side). If the source is moving in space (the normal case), the result is a compression of the space change sequence in the direction in which the source is moving (see Fig. 5, right-hand side).

Note that the speed of space change propagation remains at $c$. The consequence of the moving source is only a more-or-less compression of the gravitational changes in the direction of the movement. Figure 5 gives the impression that the propagation of space changes occurs generally in the form of waves. This arrangement is not intended in Fig. 5. However, the assumption that the space change requests are periodically issued by the quantum objects lead to a behavior that is similar to waves.

\subsection{The merge and aggregation of space changes caused by multiple sources}

To simplify the description, "multiple sources" in the following is equated to two sources. Before starting the discussion, it must be emphasized that in the causal model of spacetime dynamics, the aggregation of space changes caused by multiple sources is a dynamical process, a process that can hardly be exactly expressed by purely mathematical equations. For the overall process, three phases can be distinguished: (1) a phase in which there is no overlap/aggregation of the two space change processes, (2) a phase in which the space changes caused by the two sources partly overlap, and (3) a phase in which the space changes completely overlap. Phase (3) is reached only in the limit $t \rightarrow \infty$ (i.e., never or only with suitable approximations). Only in phase (3) would the classical physics treatment based on the center-of-mass be correct. At phase (1), the changes from the two sources propagate independently in the way described in Sec. 5.1. When the two separate areas of propagation meet, phase (2) starts. Two different cases requiring different treatments may be distinguished (see Fig. 6). In case (a), the areas meet at time step $t_{i}$ at position $x_{i}$. At time step $t_{i-1}$, the boundaries of the two areas were at position $x_{i-1}$ and position $x_{i+1}$, respectively. In case (b), the areas meet at time step $t_{j}$ when area 1 has reached 


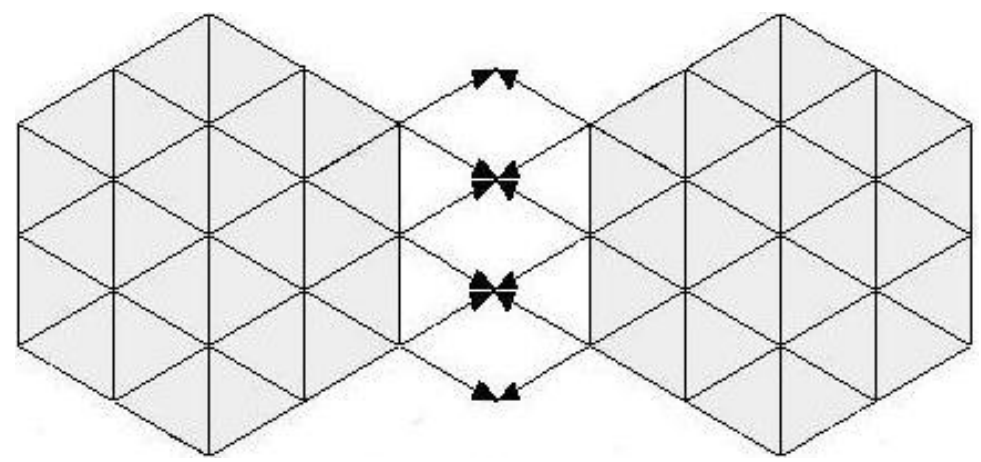

(a)

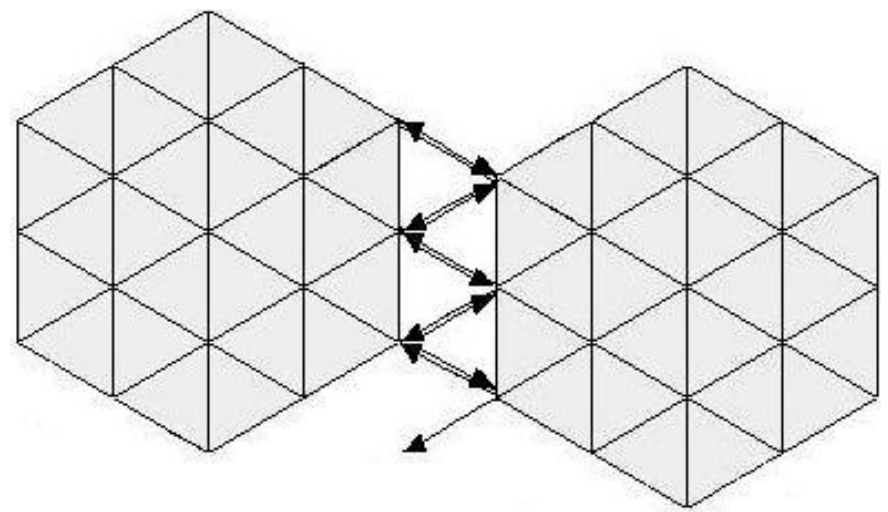

(b)

Fig. 6. Merge and aggregation of space changes caused by multiple sources.

position $x_{j}$ while area 2 has already reached position $x_{j+1}$. Case (a) fits into the model described in Sec. 5.1, specifications (5) and (6) for the single source propagation. Case (b) represents the undesirable situation where both propagatefromto $\left(s p_{i}, s p_{k}\right)$ and propagatefromto $\left(s p_{k}, s p_{i}\right)$ are requested to occur at the same time step $t_{j}$. An adaptation of specification (6) is required to suppress the mutual propagation:

$$
\begin{aligned}
& \text { propagatefromto }\left(s p_{i}, s p_{k}\right):=\{ \\
& \text { IF (propagationapplicable }\left(s p_{k}, s p_{i}\right) \\
& \text { supresspropagatefromto }\left(s p_{k}, s p_{i}\right) ; \\
& \text { normal continuation, see }(6) \\
& \}
\end{aligned}
$$

The further common phase (2) propagation continues according to the algorithm specifications (5) and (6). 


\section{Quantum Objects as Elementary Units of Causality and Locality}

In, Ref. 1 quantum objects are described as "the elementary units of causality and locality". In the context of a causal model of spacetime dynamics, the subject of this paper, quantum objects are the elementary sources of space dynamics.

As described in, Ref. 1 the following three properties distinguish quantum objects from other objects that typically occur in physics:

(1) Quantum objects are composed of multiple alternative paths with associated probability amplitudes. With the interactions (including the measurements), the multiple paths may be reduced to a single path.

(2) Quantum objects may consist of multiple spatially separated particles.

(3) Quantum objects have global attributes that apply to all of the paths and particles of the quantum object.

The combination of these three properties make quantum objects special within physics.

As shown in Fig. 7, a quantum object may be viewed as having a 2D structure. One of the dimensions represents the collection of quantum object elements, which typically consists of 1 to $n$ particles.

quantum object :=

global-quantum-object-attributes;

particle[1],

...

particle $[n]$;

In the second dimension, the quantum object consists of the set of alternatives that may be selected during the evolution of the quantum object, for example, by a measurement. In this paper, these alternatives are called "paths".

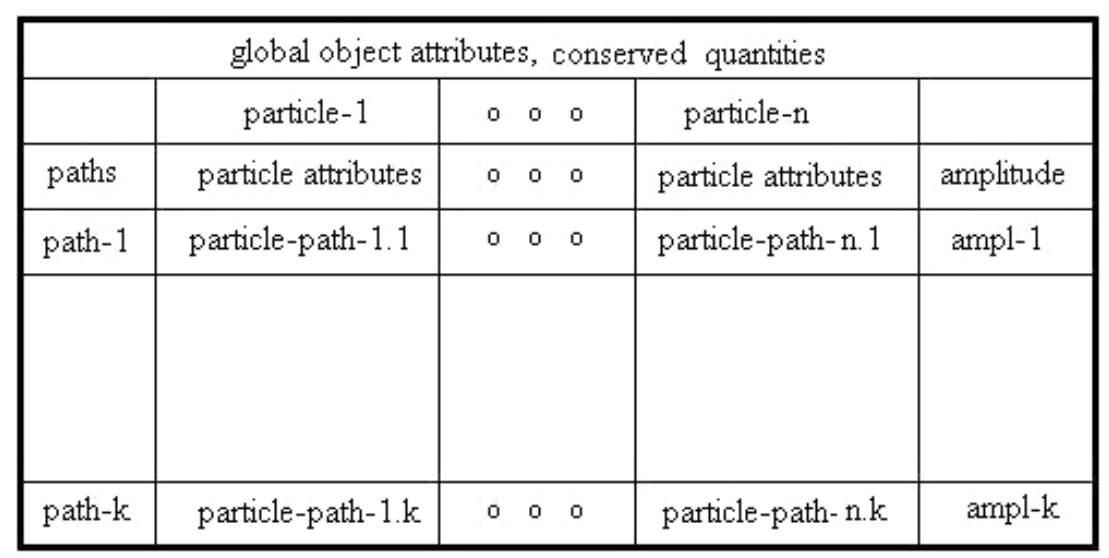

Fig. 7. Structure of the quantum object consisting of two entangled particles. 


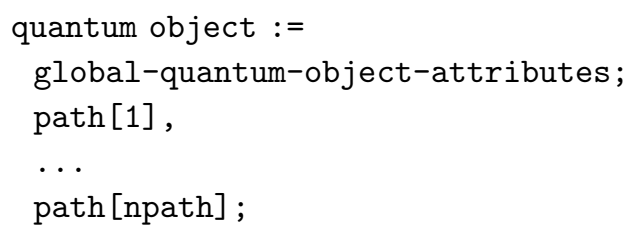

The $2 \mathrm{D}$ structure is supplemented by global attributes, i.e., attributes that apply to the complete quantum object.

Basically, two types of quantum objects can be distinguished:

- A single particle

A single particle constitutes the simplest type of quantum object. The idea of representing a particle by a set of paths was introduced by Feynman (see Ref. 13) with the formulation of quantum electrodynamics (QED).

- Collections of particles

Collections of particles that can be described by a common wave function where only specific attribute combinations can occur as measurement results represent a quantum object. Thus, the particle collection is represented by a set of paths, and each path contains the attribute combinations for all of the particles and an associated probability amplitude (see Fig. 7). Arbitrary particle collections whose common wave function would be the product of the individual wave functions do not constitute quantum objects. (As a consequence, considering the whole universe as a single large quantum object would not be in accordance with the definition of the term quantum object given in this paper.)

Composite objects such as hadrons, nuclei, and atoms that are built from (elementary) particles represent a quantum object, also.

In addition, special types of quantum objects are introduced for the causal model of interactions between quantum objects (see Ref. 2 and Sec. 7).

Quantum objects run autonomously with system-state update frequencies based on their local proper times and with either no or minimal dependency on external parameters. This arrangement means that it is possible to construct a causal model for the internal dynamics that reflects the laws of QFT. The autonomy of quantum objects ends when the quantum object interacts with another quantum object or with the space. Interactions between quantum objects are addressed in Sec. 7 and in Ref. 1.

\section{QFT in Curved Discrete Spacetime}

In the preceding sections, a model of spacetime dynamics was described where the complete spacetime dynamics was triggered by quantum objects contained in space. The internal structure and the internal dynamics of the quantum objects did not need to be examined. Although the quantum objects special role is essential for the model described, it may be also useful to apply part of the concepts of the spacetime 
model to the structure and dynamics within the quantum objects, at least to quantum objects that consist of multiple particles. The interrelations among particles is the domain of QFT. Similarly to most areas of physics, QFT requires a model of space and time as its base. For standard QFT, the continuous four-dimensional Minkowski spacetime represents that base. In the following, whether or not the curved discrete spacetime presented in the preceding sections may also offer a suitable (or perhaps even better) base for QFT is analyzed.

QFT in curved (non-discrete) spacetime has been studied extensively during the past two decades. An excellent overview is given in Ref. 14. In this section, how QFT may be mapped to curved discrete spacetime is analyzed, specifically to the causal model of curved discrete spacetime presented in the preceding sections.

\subsection{Quantum fields}

The major subject of QFT is fields. In Sec. 3, the overall system state of the causal model of curved discrete spacetime is specified, but the fields do not explicitly appear as part of the system state. Fields are described as being properties of the spacecontent component of spacepoints (see specification (3)):

$$
\begin{aligned}
& \text { spacepoint }:=\{\text { connections, spcurvature, spacecontent }\} \\
& \text { spacecontent }:=\left\{\Psi_{1}, \Psi_{2}, \ldots, \Psi_{n}\right\}
\end{aligned}
$$

The basic dynamics of the fields of QFT are assumed to be compatible with the dynamics of spacetime, as described in Sec. 5. Therefore, the fields propagate in space together with the propagation of space curvature changes described in Sec. 5 . However, the fields have additional dynamics overlaying the dynamics of space.

The primary type of field-specific dynamics is given by the equation of motion for the respective field. The simplest relativistic fields (such as the electro-magnetic field), satisfy the equation of motion (i.e., field equation, see, for example in Ref. 15)

$$
\left(\frac{1}{v^{2}} \frac{d^{2}}{d t^{2}}-\frac{d^{2}}{d x^{2}}\right) \psi(x, t)=0
$$

The $3 \mathrm{D}$ version is

$$
\left(\frac{1}{v^{2}} \frac{\partial^{2}}{\partial t^{2}}-\nabla^{2}\right) \psi(x, t)=0 .
$$

Depending on the particular context, the equation may be varied or extended by setting the right-hand side not equal to 0. For example, in, Ref. 16 the equation of motion for class 1 waves is

$$
d^{2} \psi / d t^{2}-c_{w}^{2} d^{2} \psi / d x^{2}=(2 \pi \nu)^{2}\left(\psi-\psi_{0}\right) .
$$

For a causal model, the equation of motion must be transformed to a form that is suitable for a causal model with discrete space and time. This requires translation of derivatives to " $\Delta$-units" and explicit inclusion of derivatives of time (e.g., $d \psi / d t$ ) in 
the system state (see Sec. 2). In Ref. 3, the translation of the standard equation of motion to a form that is suitable for a causal model with discrete space and time is shown for the example CA. The inclusion of the derivatives of time in the system state can be accomplished by extending specification (8) to become

$$
\text { spacecontent }:=\left\{\left\{\Psi_{1}, \Delta . \Psi_{1}\right\},\left\{\Psi_{2}, \Delta . \Psi_{2}\right\} \ldots,\left\{\Psi_{n}, \Delta . \Psi_{n}\right\}\right\}
$$

The $\Delta . \Psi_{i}$ are similar (in purpose and function) to the $\Delta . c u r v a t u r e$ components described in Sec. 5.1. ${ }^{\mathrm{f}}$

A further type of field-specific dynamics is fluctuations. According to QFT, fields fluctuate permanently. In a causal model, the field fluctuations can be included as random events. In the proposed causal model of spacetime, the fluctuations could also be triggered by special situations that occur during the (complex) propagation of space and the associated field(s).

Path integrals and paths: In Feynmans interpretation, the probability amplitude for an event is determined by adding together the contributions of all paths in configuration space. For wave function (in position representation), the path integral formula is typically written as

$$
\psi(x, t)=\frac{1}{Z} \int_{x 0} e^{i S(x, \dot{x})} \psi_{0}(x(t)) D x,
$$

where $S$ is the action and $Z$ a normalization factor. $D x$ denotes integration over all paths.

The major applications of the path integral formulation in QFT are the propagation of probability amplitudes through space (see Ref. 13) and the computation of the outcome of particle scatterings by use of Feynman diagrams. The mapping and application of the path integral formulation to the causal model of spacetime dynamics is straightforward for the following reasons:

(1) In, Ref. 17 one advantage of the path integral formulation is described as "the path integral allows a physicist to easily change coordinates between very different canonical descriptions of the same quantum system". While this is true in general, it is particularly true for mapping to a spacetime structure that includes already existing connections (i.e., paths) between the spacepoints.

(2) The discreteness of the proposed causal model of spacetime matches naturally with the way path integrals are typically introduced in textbooks on QFT. Typically, the introduction of path integrals starts with a set of discrete paths, which then becomes continuous towards the limits $\Delta t \rightarrow 0$. No reason exists for moving to the limit $\Delta t \rightarrow 0$ unless the underlying spacetime model requires this.

\footnotetext{
${ }^{\mathrm{f}}$ Therefore, the question arises as to whether the $\Delta . \Psi_{i}$ should also be associated with the spacepoint connections.
} 
(3) A similar argument concerning discreteness applies to the limit $n$ (number of paths) $\rightarrow \infty$. If the spacetime structure limits the number of paths to a discrete finite number, a more realistic model may result. With QFT, the assumption of discreteness may eliminate the need for cut-offs and renormalization procedures.

(4) Although the path integrals of QFT are based on Minkowski space and thus support the integration of space and time of SRT, space and time play rather different roles in the path integrals, similar to the roles space and time have in the causal models described in Sec. 2.

\subsection{Particle scatterings}

The QFT predictions of the outcome of particle scatterings is a major area of QFT that led to the impressive success of QFT. Further areas of the theory that are not directly related to particle scatterings have been experimentally tested through corresponding particle scattering experiments (e.g., by the use of accelerators and colliders).

The scattering matrix and the Feynman diagrams are the major tools for QFT treatment of particle scatterings. Because it is not possible to provide in this paper, a somewhat complete introduction to QFT particle scattering, Feynman diagrams, and other concepts, nor is it possible to show mapping of these standard QFT features to the proposed causal model of spacetime dynamics, in the following, the subject is discussed in terms of an example of particle scattering.

The example used is electron-positron scattering, $\left(e^{-}, e^{+} \rightarrow e^{-}, e^{+}\right)$(called Bhabha scattering). The standard QFT computation is based on Feynman diagrams and the equations derived from the diagrams. The possible first-order diagrams for the Bhabha scattering are shown in Fig. 8. QFT provides rules that enable derivation

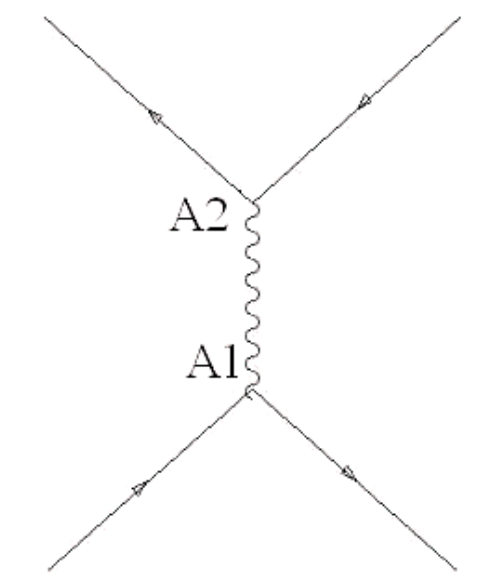

Bhabha Scattering Diagram A

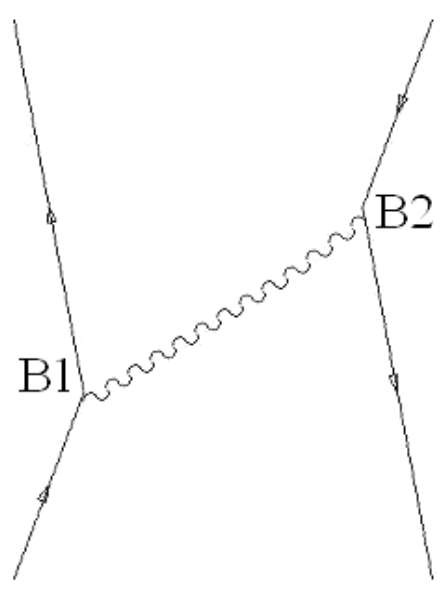

Diagram B

Fig. 8. Feynman diagrams for Bhabha scattering. 
of equations for computing the scattering matrix amplitudes from the pertinent Feynman diagrams. For Bhabha scattering, these rules result in

$$
M_{A}=(-i e)^{2} \bar{v}\left(\boldsymbol{p}_{2}, s_{2}\right) \gamma_{\mu} u\left(\boldsymbol{p}_{1}, s_{1}\right)\left(-i g^{\mu \nu} /\left(p_{1}+p_{2}\right)^{2}\right) \bar{u}\left(\boldsymbol{p}_{1}^{\prime}, s_{1}^{\prime}\right) \gamma_{\nu} v\left(\boldsymbol{p}_{2}^{\prime}, s_{2}^{\prime}\right) .
$$

and

$$
M_{B}=(-i e)^{2} \bar{u}\left(\boldsymbol{p}_{1}^{\prime}, s_{1}^{\prime}\right) \gamma_{\mu} u\left(\boldsymbol{p}_{1}, s_{1}\right)\left(-i g^{\mu \nu} /\left(p_{1}-p_{1}^{\prime}\right)^{2}\right) \bar{v}\left(\boldsymbol{p}_{2}, s_{2}\right) \gamma_{\nu} v\left(\boldsymbol{p}_{2}^{\prime}, s_{2}^{\prime}\right)
$$

According to the usual QFT notation, $u()$ represents the entry electron, $\bar{u}()$ the exit electron, $\bar{v}$ the entry positron, and $v()$ the exit positron. $M_{A}$ and $M_{B}$ are the probability amplitudes. The total probability amplitude $M$ for Bhabha scattering (firstorder perturbation) is $M=M_{A}-M_{B}$.

An actual computation for predicting the outcome of a scattering experiment can be performed either "in position space" or "in momentum space". This means that the paths shown in Feynman diagrams are interpreted as connections between positions in space or between different values of momentum. In practice, the computation in momentum space is easier and therefore generally favored. For discussion on QFT in curved discrete spacetime, the QFT position space model relative to the proposed spacetime model is of interest.

As described above for the path integral formulation, the mapping of QFT position space paths to the spacetime structure described in Secs. 4 and 5 is generally straightforward. The dispersion of space change propagation as described in Sec. 5 can be used as a base model for the many paths represented in the path integral. The splitting of paths and their reunion as occurring in the Feynman diagrams of QFT can be mapped to the network of spacepoint connections. However, this mapping of QFT paths (including Feynman diagrams) to the network of spacepoint connections does not deliver a complete causal model of QFT particle scattering. A causal model of QFT particle scattering must in addition reflect the processes that can be derived from the applicable Feynman diagrams. In Ref. 3, a causal model of QFT interactions (i.e., QFT scatterings) is described where the Feynman diagrams are translated into sequences of split() and combine() operations. The split() and combine() operations are analogous (although not equal) to the creation and annihilation operators of QFT. In a causal model, including spatial positions (i.e., a position space model), such as the presently discussed model, the split() and combine() operations must be assigned to positions in space. In Fig. 8, the respective positions are labeled A1, A2, B1, and B2. QFT defines the types of possible split() and combine() operations. For QED, the possible operations are (see, for example [Ref. 18, p. 111]):

$$
\begin{aligned}
& \text { for split }(): e^{-} \rightarrow e^{-}+\gamma ; e^{+} \rightarrow e^{+}+\gamma ; \gamma \rightarrow e^{+}+e^{-} \text {. } \\
& \text { for combine }(): e^{+}+e^{-} \rightarrow \gamma ; e^{-}+\gamma \rightarrow e^{-} ; e^{+}+\gamma \rightarrow e^{+} .
\end{aligned}
$$

When there exist multiple potential positions for a specific in-configuration and outconfiguration (which is generally the case), this leads to multiple possible "paths" whose probability amplitudes must be summed up by the path integral. For the spacetime structure proposed in Secs. 3 and 4 with the interconnected spacepoints, 

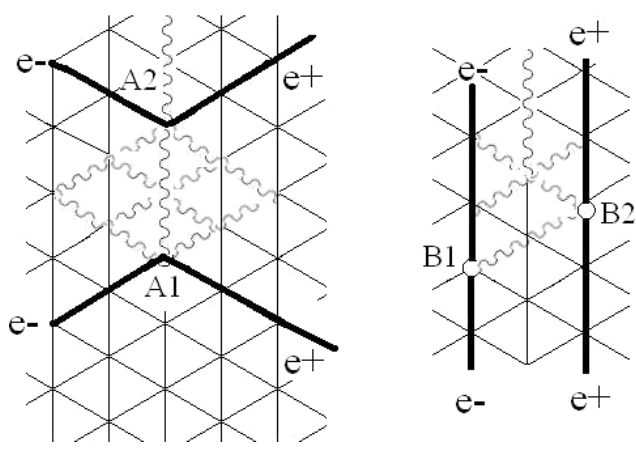

Fig. 9. Split/combine points mapped to space.

the split/combine points A1, A2, B1, and B2 must be assigned to spacepoints. The spacepoints of the proposed spacetime structure are especially suited to the association of split/combine points because each spacepoint already has associated connections. Thus, the initial assumption is: each spacepoint is a potential split/combine point. This is also the assumption in standard QFT. As we are discussing QFT in the context of a special model of spacetime, it is worth analyzing whether this very general assumption (that each spacepoint is a potential split/combine point) can be narrowed by a plausible causal model. The causal model must also specify how the actual split/combine points are determined and mapped to the spacepoint connections.

\subsection{Causal model of QFT scatterings in curved discrete spacetime}

Diel in Refs. 1 and 2 describe a causal model of QT/QFT, with focus on QFT interactions, such as QFT scatterings. In these papers, the overall interaction process is described. However, the relation to the propagation of spatial system state changes is not addressed. The focus of the present section is the mapping of split/combine points to spacepoints and their connections in conjunction with the propagation of particles.

In the causal model of QFT scatterings in curved discrete spacetime, each spacepoint involved in particle propagation is a split/combine point (potential as well as actual). Compatibility with the meaning of the applicable Feynman diagrams is achieved by the spacepoints different configurations with respect to their in/out connections and the content $\Psi$ and $\Delta . \Psi$ is associated with the spacepoint. The spacepoints involved in propagating a particle typically have 12 connections to neighbor spacepoints. At a specific step in particle propagation, the spacepoint connections can be grouped into (1) in-connections, (2) out-connections and (3) possibly connections that are neither in-connections nor out-connections (see Fig. 9). At least one of the in-connections and one of the out-connections is dynamically determined to be a "primary connection", which means it supports the particle type of the propagating particle. The remaining in-connections and outconnections are secondary connections. Secondary connections are always boson 
connections, e.g., photon connections with QED. ${ }^{\mathrm{g}}$ The assignment of the connection attributes (in-connection/out-connection, (virtual) particle type) is performed as part of a dynamical process, the particlepropagation() process.

particlepropagation $\left(\right.$ particle $\left.p_{i}\right):=\{$

FOR (all spacepoints $s p_{j}$ assigned to particle $\left.p_{i}\right)\{$

priminconn $\leftarrow$ determineprimaryinconnection () ;

iaobject $\leftarrow$ derivefrom(priminconn.source);

FOR (all connections $s p_{j}$.connection con $_{j}\{$

IF $\left(s p_{j}\right.$. connection $\left.{ }_{j} \cdot \Delta . \Psi>0\right)$ iaobject $\leftarrow$ combine(iaobject, $s p_{j}$. connection $\left._{j}\right)$;

\}

outconns $\leftarrow$ determineoutconnections $\left(s p_{j}\right.$, iaobject $)$;

primoutconn $\leftarrow$ determineprimaryoutconnections(outconns);

propagatefromto(iaobject, primoutconn.target);

FOR (all outconns outconn $\left.n_{k}\right)\{$

propagatefromto(iaobject, outconn $\left.n_{k}\right)$;

\}

\}$\}$

As can be seen, the primary parameters that determine the propagation of a particle are the spacepoint connections and the associated space content.

spacecontent $:=\left\{\left\{\Psi_{1}, \Delta . \Psi_{1}\right\},\left\{\Psi_{2}, \Delta . \Psi_{2}\right\} \ldots,\left\{\Psi_{n}, \Delta \Psi_{n}\right\}\right\}$ (see specification (10) in Sec. 7.1). From the spacecontent, the $\Delta . \Psi_{i}$ determine the direction of propagation.

The above specification (15) shows normal propagation of a particle, including the possible decay of the particle and virtual particle fluctuations. However, it does not show the complete interaction and scattering between two particles. The special case "scattering" is recognized when the function "derivefrom(priminconn.source)" detects that the source of the primary in-connection is a spacepoint belonging to another quantum object. When this is recognized and further conditions are satisfied, the normal particle propagation process specified in (15) is abruptly terminated and the scattering process is started. The sudden termination of the propagation of both particles involved in the scattering is equivalent to what is usually referred to as collapse of the wave function in the literature. In terms of the causal model, the collapse of the wave function means that the two physics engines associated with the two quantum objects are terminated. Instead, a new quantum object, called "interaction-object", with a new physics engine is started at the spacepoint where the interaction occurred.

Diel $^{1,2}$ describe this process in more detail in a more general context. Particle scatterings are considered special types of the more general "QFT interactions". QFT interactions play a crucial role with QT measurements (see Ref. 19). The

g In Fig. 9, only some examples of possible secondary connections (i.e., photons) are shown. 
further processing of the particle scattering (more generally of a QFT interaction) is performed as progression of the interaction-object.

progressinteractionobject(iaobj) $::=\{$

FOR (all virtualparticles $v p_{j}$ of the interaction - object iaobj) \{

vparticlepropagation $\left(v p_{j}\right)$;

\}

vparticlepropagation() is identical to (15) particlepropagation() except for a small difference when two (virtual) particles interact at a given spacepoint. As described above, when two quantum objects interact, the annihilation of the two quantum objects occurs and an interaction-object is created. Then, the physics engines of the two quantum objects are terminated. When two virtual particles that are part of the same quantum object interact, the two virtual particles are annihilated and a new virtual particle is created. However, the common physics engine of the persistent quantum object is not abandoned.

The above-described causal model of QFT scatterings in curved discrete spacetime leads to the fundamental processes of QFT (for example, the QED processes mentioned in specification (14)) that occur in Feynman diagrams. Whether or not the overall propagation process that consists of a sequence of these fundamental processes results in the same final probability amplitude is yet to be verified. A possible verification that accounts for the discreteness and curvature of spacetime apparently requires computer simulations ${ }^{\mathrm{h}}$ and use of Monte Carlo procedures.

\subsection{The internal dynamics of quantum objects}

The internal dynamics of quantum objects refers to quantum objects consisting of multiple particles. According to Sec. 6, these are quantum objects in bound systems and the interaction object. The interaction-object has been described in Sec. 7.2 as the quantum object that processes QFT interactions, such as particle scatterings through the internal interactions among the particles (including virtual particles) that constitute the quantum object. The internal interactions have been mapped to spacepoints and their connections that establish dynamically changing paths.

\section{Discussions}

\subsection{Major findings from the causal models of spacetime dynamics}

The constraint (originating from SRT/GRT) that changes can propagate to distant locations only through a series of neighboring locations, necessitating a detailed model of the propagation of space (curvature) changes. In the model of spacetime dynamics described in Secs. 4 and 5, the propagating spacetime changes have at each

${ }^{\mathrm{h}}$ Computer simulations are in process with the author. 
stage of the process a well-defined direction. Care must be taken so that the propagation of space changes that originate from multiple distributed sources do not result in loops, crossings and other obscure situations. This has resulted in a model where the combined propagation of the space changes start when the individual propagations meet at specific events in spacetime. From this point, the combined accumulated propagation from the multiple sources continues away from the nonaccumulated areas. This may be considered to be the development of areas (islands) of "non-accumulated gravitational curvature changes". This may lead to interesting consequences, both at the micro level (i.e., QFT) as well as at the macro level (i.e., cosmology). However, these are not further discussed in this paper.

In the second part of the paper (Sec. 7), a rough mapping of quantum field theory to the proposed model of spacetime dynamics is described. These considerations also apply to the internal dynamics of quantum objects. The mapping of the position space model of QFT to the interconnected spacepoints of the proposed spacetime model opens the opportunity for a more general model of virtual particle creation/ annihilation as expressed in Feynman diagrams. A spacepoint may be the target of multiple virtual particle annihilations and the source of multiple virtual particle creations.

\subsection{The value and need for causal models}

The model of spacetime dynamics is described in the form of a causal model, i.e., in terms of a model of the system state and a process consisting of a succession of state transitions under various conditions. The model of spacetime dynamics has been described as a causal model, because

(1) The main purpose of causal models is the specification of the relationship of the progression of time to the changes in the system state including space.

(2) Causal models are best suited to specifying non-trivial dynamics.

(3) If the causal model is formulated according to the formal model described in Sec. 2 (i.e., with a complete specification of the system state and a complete specification of the possible state transitions) this enforces the completeness of the overall model.

\subsection{The value and need for discrete units of time and space}

For the development of a model of an area of physics, such as the model of spacetime dynamics, the need for the assumption of discrete units of time and space depends entirely on the domain and the aspects that are addressed by the model. If the model focuses on aspects where the granularity of the major parameters can be assumed to be sufficiently fine, such that calculations by use of differentials and integrals are applicable, there is no need to think about discrete smallest units of time and space. In contrast, if the model addresses aspects where the granularity matters, the use of 
discrete parameters is inevitable. More concretely, discrete units of time and/or space should be assumed for models that address the following:

(1) The very low level of detail with respect to time, space and/or other parameters of the system state (under the assumption that there is a non-zero lowest level of detail).

(2) Processes with abrupt (i.e. non-differentiable) state changes (the "collapse of the wave function" is an example),

(3) Causality and simultaneity based on uniform time intervals at a low level of detail.

(4) Processes leading to singularities.

For the model of spacetime dynamics described in the preceding sections all the above listed reasons for the assumption of discrete units of time and space are applicable (item (4) is not addressed in the present paper, but is a candidate for future work). In addition, the causal model described in Sec. 2 aims for maximum generality, which means that all the above listed cases should be supported.

\subsection{Related work}

The proposed "model of spacetime dynamics with embedded quantum objects" may be viewed as an attempt to specify the elementary structure of spacetime such that the major laws of both SRT/GRT and QT/QFT are satisfied. A number of works towards the same or similar goal have been published. The works that show the most similarities with the model described in this paper in terms of the overall orientation (background independence, discreteness of time, space and paths, expressing causal relationships) are the loop quantum gravity (see Refs. 20, 21) and its layers causal dynamical triangulation (CDT) (see Ref. 11) and spin foam networks (see Ref. 22). The spacetime structure of the model described in this paper is based on CDT. However, it was felt that adaptations were required to further refine the causal relationships of spacetime dynamics (see Sec. 4).

Another effort that addresses the elementary structure of spacetime and where certain similarities can be observed is the work towards the fractal structure of spacetime (see Ref. 23). Looking, for example, at Fig. 9 and imagining levels of structural refinement and perturbation leads to fractal-like structures. However, this apparent similarity is rather superficial. Further investigations are required to exploit the feasibility and potential value of an adaptation towards a fractal-based spacetime structure.

The work known by the name "causal fermion systems" (see Ref. 24) shows the commonality with the subject of this paper insofar as in both approaches the emergence of spacetime is coupled with the emergence and dynamics of particles (i.e., quantum objects).

A further approximation between the causal model of QT/QFT described and referenced in this paper and some of the concepts mentioned above may be reached when the model described in Sec. 7 ("QFT in curved discrete spacetime") will be further refined. 


\section{Conclusions}

The main conclusion from the work described in this paper is that it requires a causal model to describe the dynamics of a complex area of physics, such as GRTcompatible spacetime. The second equally important finding is that pure GRT is not a sufficient base for deriving a complete and detailed causal model of spacetime dynamics. Additional "design decisions" must be made that specify details of the physics that cannot be derived from GRT.

As a follow-on conclusion, causal models (i.e., models of the dynamics of something) that aim to specify causal relationships at a low level of detail have been found to apparently tend towards requesting discrete units of time and space (and possibly further components of the system state).

For the construction of the model of spacetime dynamics, the key assumption has been that spacetime dynamics and spacetime curvature as defined by GRT must already start at the micro level of physics and has significant implications already at the micro level. In contrast to other approaches that have been proposed that equate to a "micro level" with theoretical limits, such as Planck scale, in the proposed model, spacetime dynamics starts at (and is triggered by) elementary objects called quantum objects. The low-level start of spacetime dynamics, the propagation of the spacetime changes in terms of discrete units of space and time, and the necessity for a causal model of the accumulation of spacetime changes caused by multiple sources has resulted in a highly dynamic causal model with unexpected effects, such as "islands of non-accumulated spacetime curvature changes".

Quantum objects, the elementary sources of spacetime dynamics, are defined as the minimal objects whose internal dynamics must be described by the laws of quantum (field) theory. In the causal model of QT/QFT quantum objects are also units of simultaneity and proper time (comparable to inertial systems). This separates the areas where the laws of GRT are in force from the areas where the laws of QT are in force, while at the same time the coexistence of the two areas of physics becomes visible.

\section{References}

1. H. Diel, Quantum objects as elementary units of causality and locality, arXiv:1609.04242v1.

2. H. Diel, A functional model of interactions in quantum theory, arXiv: 1405: 6099v2.

3. H. Diel, A lagrangian-driven cellular automaton supporting quantum field theory, http:// arxiv.org/abs/1507.08277.

4. H. Diel, The completeness, computability, and extensibility of quantum theory, arXiv:1512.08720.

5. S. Wolfram, A New Kind of Science (Wofram Media, UK, 2002).

6. A. Ilachinski, Cellular Automata: A Discrete Universe (World Scientific, Singapore, 2001).

7. G. 't Hooft, The Cellular Automaton Interpretation of QM (Springer, New York, 2016).

8. Wikipedia Causal Dynamical Triangulation, https://en.wikipedia.org/wiki/Causal_ dynamical_triangulation (2017).

9. R. Loll, J. Ambjorn and J. Jurkiewicz, Reconstructing the Universe, arXiv:hep-th/0505154. 
10. J. Ambjorn, J. Jurkiewicz and R. Loll, Quantum gravity, or the art of building spacetime, arXiv:hep-th/0604212.

11. R. Loll, J. Ambjorn and J. Jurkiewicz, The Universe from Scratch, arXiv:hep-th/0509010.

12. B. F. Schutz, A First Course in General Relativity (Cambridge University Press, New York, 2009).

13. R. P. Feynman, QED: The Strange Theory of Light and Matter (Princeton University Press, US, 1985).

14. K. Fredenhagen and T.-P. Hack, Quantum field theory on curved spacetime and the standard cosmological model, in The Message of Quantum Science, eds. P. Blanchard, J. Froehlich (Springer Verlag, Berlin Heidelberg, 2015).

15. C. W. Wong, Introduction to Mathematical Physics, Methods $\&$ Concepts (Oxford University Press, Oxford, 1991).

16. M. Strassler, Conversations About Science with Theoretical Physicist, http://profmattstrassler.com/articles-and-posts/particle-physics-basics/Higgs/ (2017).

17. Wikipedia on Path integral formulation.

18. F. Mandl and G. Shaw, Quantenfeldtheorie (AULA Verlag, Germany, 1993).

19. H. Diel, A model of the measurement process in quantum theory, arXiv:1405:5982v1.

20. T. Thiemann, Loop quantum gravity: An inside view, Approaches to Fundamental Physics, Lecture Notes in Physics, Vol. 721, pp. 185-263.

21. C. Rovelli, Loop quantum gravity, http://www.livingreviews.org/lrr-1998-1, Living Rev. Rel. 1 (1998) Retrieved 13 March 2008.

22. J. C. Baez, Spin foam models, https://arxiv.org/gr-qc/9709052v3.

23. Y. Tao, Quantum behavior arises because our universe is a fractal, Rep. Adv. Phys. Sci. 1 (2017) 1750006, doi: 10.1142/S2424942417500062.

24. F. Finster and J. Kleiner, Causal fermion systems as a candidate for a unified physical theory, J. Phys. Conf. Ser. 626 (2015) 012020, http://adsabs.harvard.edu/abs/2015JPhCS.626a2020F, arXiv:1502.03587, https://arxiv.org/abs/1502.03587, doi: 10.1088/1742-6596/626/1/012020. 